\title{
Special session of the XMEGA group
}

\author{
Michael F. Corcoran ${ }^{1,2}$, Julian M. Pittard ${ }^{3}$, Sergey V. Marchenko ${ }^{4}$, \\ and the XMEGA group ${ }^{5}$ \\ ${ }^{1}$ Laboratory for High Energy Astrophysics, \\ NASA Goddard Space Flight Center, Greenbelt, MD 20771, USA \\ ${ }^{2}$ Universities Space Research Association, \\ 7501 Forbes Blvd, Ste 206, Seabrook, MD 20706, USA \\ ${ }^{3}$ School of Physics and Astronomy, University of Birmingham, \\ Birmingham B15 2TT, England \\ ${ }^{4}$ Département de Physique, Université de Montréal, \\ CP-6128, Succ. Centre-Ville, Montréal, Qc H3C 3J7, Canada \\ ${ }^{5}$ http://lheawww.gsfc.nasa.gov/users/corcoran/xmega/xmega.html
}

\begin{abstract}
On the day prior to the Symposium, we held a special session to review the current status of the XMEGA observations, to discuss upcoming observations, and to plan for future observing programs
\end{abstract}

\section{Introduction}

The 'XMEGA' campaign was organized at the 'Colliding Winds in Binary Stars' Workshop, November 1995, La Plata (Niemela \& Morrell 1996). It includes scientists interested in the phenomena of X-ray emission from hot stars. We have tried to develop detailed X-ray observing programs, coordinated with ground based observations, for a number of important systems.

\section{WR 140}

The XMEGA group has used $A S C A$ to monitor the colliding wind X-ray emission from WR140, a long-period, massive binary (WC7+O4-5, $P=7.9 \mathrm{y}, e=0.85$ ). These X-ray results are discussed by Pollock et al. elsewhere in these Proceedings.

Coordinated optical observations show: (i) significant rise in the emissivity of the lines with relatively low ionization potentials (He I and C III ), taking place precisely at periastron passage; and (ii) 0.03 mag minimum in the optical light curve occurring 0.05 (phase) after periastron passage and probably related to dust formation.

\section{3. ŁOri}

We have used ASCA to monitor $\iota$ Ori (O9III+B1III, $P=29.13 \mathrm{~d}, e=0.76$ ), a system which should show wind-collision X-ray emission (perhaps modulated 
by radiative braking). Contrary to WR $140, \iota$ Ori shows no signs of wind-wind interaction in the optical light curve, and only subtle line profile variability which might be attributed to a wind-wind collision.

Iota Orionis was twice observed with $A S C A$ during 1997, once at periastron and once at apastron. The most surprising result is that the $\mathrm{X}$-ray emission is remarkably constant. One plausible explanation is that there is a large contribution to the overall X-ray emission from intrinsic shocks in the stellar wind of the primary. This emission would not be strongly affected by the orbital separation, thus reducing the overall variation. A more tentative explanation suggested by preliminary hydrodynamical models relies on the wind momenta of the stellar winds being so unbalanced that the colliding wind shock is collapsed onto the photosphere of the secondary throughout the entire orbit. Both of these interpretations are also supported by the constancy of the characteristic temperatures from Raymond-Smith model fits to the data at periastron and apastron.

Iota Orionis is an interesting system from the viewpoint of the radiative braking paradigm. The system could span three important regimes: wind collision with the photosphere at periastron, wind-wind collision primarily due to ram balance at apastron, and a wind-wind collision controlled by radiative braking at intermediate separations.

\section{The Carina nebula}

We were awarded $300 \mathrm{ksec}$ of time critical ROSAT time primarily to obtain a lightcurve of HD 93205, perhaps the most massive colliding wind binary (next to $\eta$ Car?), and also to measure variability in $\eta$ Car and other bright sources, and extend the X-ray luminosity function. We obtained a $50 \mathrm{ksec}$ observation in January 1998, which enabled us to spatially resolve the variable source in $\eta$ Car. Unfortunately, the ROSAT mission ended prior to obtaining the remainder of the observing time. (The end of the ROSAT mission in September 1998 also resulted in the loss of another XMEGA program to measure the X-ray lightcurve of HD 159176.)

\section{Future projects}

We have been awarded a $75 \mathrm{ksec}$ pointing at the starburst-like region NGC 3603 with the ACIS instrument on the Chandra X-ray Observatory ( $C X O$, formerly $A X A F)$. The purpose of this observation is to obtain spectra and fluxes for highmass members of this cluster. We have a number of other ideas for additional observing programs. Those interested in joining the XMEGA group should email Mike Corcoran ( corcoran@barnegat.gsfc.nasa.gov).

\section{References}

Niemela, V.S., Morrell, N.I. (eds.) 1996, Colliding Winds in Binary Stars, Proc. Workshop celebrating the 80th birthday Jorge Sahade, RevMexAA Ser. Conf. 5. 